\title{
Microbiota's 'little helpers': bacteriophages and antibiotic-associated responses in the gut microbiome
}

\author{
Christine L Sun ${ }^{1}$ and David A Relman 1,2,3*
}

\begin{abstract}
Antibiotics alter the abundance and types of

bacteriophage-associated genes in the mouse gut, suggesting that phage help bacterial communities during times of stress.
\end{abstract}

\section{When the going gets tough ...}

Disturbances of the human microbiota and their gene pool, the microbiome, seem to be an increasingly common global feature of modern life. Drugs and microbial pathogens are among the most widespread and insidious causes of microbiota disturbance, but antibiotics deserve special attention, not only because of their pervasive effects on the indigenous human microbiota [1], but because of the threats posed by the growing spread of antibiotic resistance. Genes responsible for antibiotic resistance have been found even in 'pristine' environments [2], such as in soil from a remote Alaskan site, as well as in the human microbiome itself [3]. In fact, the use of antibiotics can promote the mobilization and exchange of resistance genes. Resistance genes are usually carried by mobile genetic elements, such as plasmids, transposons and phage. The majority of phage in the human gut as well as those in other mammalian intestinal tracts are probably temperate, and therefore spend much of the time as integrated prophages [4]. It is well established from environmental studies that phage shape bacterial community structure and function via predation and gene transfer [5]. Temperate phage often contain bacterial host genes and spread novel or functionally important genes throughout the host population. Phageencoded host genes can increase the fitness of the

\footnotetext{
*Correspondence: relman@stanford.edu

'Department of Microbiology \& Immunology, Stanford University School of Medicine, Stanford, CA 94305-5124, USA

Full list of author information is available at the end of the article
}

bacterial host, which ultimately improves the fitness of the phage.

Many of the factors responsible for human microbial ecosystem disturbance produce conditions of oxidative stress for cells of both host and microbe. For example, many bactericidal antibiotics kill via production of hydroxyl radicals in both Gram-positive and Gramnegative bacteria [6]. Likewise, pathogens typically induce inflammation and reactive oxygen species in the host, and also tend to compete especially well with commensals under these conditions. Interestingly, horizontal gene transfer occurs preferentially under exactly these conditions. It has been shown that beta-lactam antibiotics trigger the bacterial SOS response, induction of prophage in Staphylococcus aureus and phage-mediated transfer of pathogenicity genes [7]. Similarly, quinolone antibiotics induce Shiga toxin-encoding prophage in mice infected with lysogenic enterohemorrhagic Escherichia coli, enhanced production of toxin, transfection of E. coli nonlysogens within the gut and greater mortality of the host [8], with supporting data from studies of sick children. Under conditions of gut inflammation associated with salmonellosis, transconjugation of plasmids between Salmonella and E. coli occurs at much higher rates than under normal conditions [9]. Thus, horizontal gene transfer is effected by ecosystem disturbance, and suggests a form of adaptive response.

In this context, a recent study by Modi and colleagues in Nature [10] provides new insight into the effect of antibiotics on phage populations in the gut and the role of phage in the spread of antibiotic resistance.

\section{Phage as agents of ecosystem homeostasis and adaptation}

Modi et al. treated two groups of mice with either ciprofloxacin or ampicillin, and included untreated mice as controls [10]. Fecal matter from each group was collected after 8 weeks and DNA from purified phage communities was extracted. A total of $210 \mathrm{Mb}$ of community DNA sequence was obtained. Quantitative PCR data indicated that $<0.1 \%$ of the sequences derived from bacterial 
contamination. To date, this is the first in vivo study of the impact of antibiotics on the overall population of extracellular phage in the gut.

The study revealed an increased representation of phage-encoded genes associated with antibiotic resistance after 8 weeks of exposure to each of the two antibiotics compared with the control groups. One assumes that these resistance genes were present in bacterial genomes, perhaps in prophage, or within extracellular phage, prior to antibiotic treatment. In addition to genes that confer resistance to ciprofloxacin or ampicillin, there was enrichment of genes that confer resistance to other members of these two classes of antibiotics, as well as genes associated with resistance to other classes of antibiotics, providing evidence of coinheritance of heterologous drug resistance. Antibiotic treatment also led to an enrichment of genes related to metabolic processes that might mediate the killing effects of these drugs (such as oxidative stress), or to beneficial, adaptive responses that might mitigate these effects (such as DNA repair) or enhance glycan degradation and other metabolic capacities of the community [10]. The authors then transfected pools of fecal bacterial isolates from untreated mice with phage purified from the feces of treated mice, and found that the fraction of antibioticresistant isolates increased more than it did using phage from untreated mice, thereby demonstrating that the phage-encoded antibiotic resistance genes were functional. An analysis of the inferred interactions between phages and bacterial hosts suggests that antibiotic treatment increases the numbers and kinds of interactions, and hence the genetic potential of individuals and consortia within the bacterial communities.

There are some unresolved questions with regard to this study. The increase in phage-encoded host genes might result either from blooms in pre-existing antibiotic-resistant lysogenic bacteria, with subsequent phage induction, packaging of host DNA and release of phage, or from primary induction of resistance-encoding prophage and subsequent phage propagation, or both. To disentangle these different mechanisms, one might sequence the corresponding bacterial host populations, in addition to the phage pools, in order to characterize intrinsic resistance genes, the genomes of integrated phage and relative abundances of the hosts, before and after antibiotic treatment. In addition, the role of other extrachromosomal elements in this setting is unclear. Comprehensive assessments of the microbial cell-associated and cell-free metagenomes before and after treatment would help address the contribution of plasmids and transposable elements to the response to disturbance and ecosystem adaptive capacity. From an evolutionary perspective, phage populations confer flexibility and adaptive capacity upon the microbiota; however, in considering the significance of the findings in this study we must bear in mind that the concentrations of antibiotics used here and on humans and other hosts today exceed by many orders of magnitude the concentrations that have been encountered by naturally occurring microbial communities over evolutionary time scales.

\section{In pursuit of further understanding}

The findings from the study by Modi et al. lend powerful support to the well-established role of bacteriophages in horizontal gene transfer within complex naturally occurring microbial communities, and in particular, within the human microbiome. The enrichment of phage-encoded antibiotic resistance genes as well as other beneficial host genes following antibiotic exposure suggests that phage are key factors in the spread of antibiotic resistance. Examination of complete bacterial and phage genomes in control and treated animals, especially humans, would help elucidate the roles of phage in shaping and reshaping the composition and structure of gut microbial communities before and after various forms of disturbance. Future studies would also benefit from more frequent sampling of individual animals before and after initiation of antibiotic exposure, as well as shorter drug exposures, lower doses, and the use of antibiotics that do not induce phages. These study features would enable a more detailed appreciation of microbial community temporal dynamics, network interactions, community stability and resilience, and variation in these microbiome characteristics among different hosts. The longer term goal of such work is a predictive understanding of ecosystem responses and functions, such that health can be maintained in the face of perturbations and that targeted interventions can be designed to treat or prevent disease-associated community disturbances.

\section{Competing interests}

The authors declare that they have no competing interests.

\section{Acknowledgements}

DR was supported in part by the National Institutes of Health, grants DP1OD000964, R01-Al092531 and R01-GM099534, a Distinguished Clinical Scientist Award from the Doris Duke Charitable Trust, and by the Thomas C and Joan M Merigan Endowment at Stanford University.

\section{Author details}

'Department of Microbiology \& Immunology, Stanford University School of Medicine, Stanford, CA 94305-5124, USA. ²Department of Medicine, Stanford University School of Medicine, Stanford, CA 94305-5107, USA. ${ }^{3}$ Veterans Affairs Palo Alto Health Care System, Palo Alto, CA 94304-1207, USA.

Published: 30 July 2013

\section{References}

1. Dethlefsen L, Relman DA: Incomplete recovery and individualized responses of the human distal gut microbiota to repeated antibiotic perturbation. Proc Natl Acad Sci U S A 2011, 108 Suppl 1:4554-4561.

2. Allen HK, Donato J, Wang HH, Cloud-Hansen KA, Davies J, Handelsman J: Call of the wild: antibiotic resistance genes in natural environments. Nat Rev Microbio/ 2010, 8:251-259. 
3. Sommer MO, Dantas G, Church GM: Functional characterization of the antibiotic resistance reservoir in the human microflora. Science 2009, 325:1128-1131

4. Reyes A, Haynes M, Hanson N, Angly FE, Heath AC, Rohwer F, Gordon Jl: Viruses in the faecal microbiota of monozygotic twins and their mothers. Nature 2010, 466:334-338.

5. Chibani-Chennoufi S, Bruttin A, Dillmann ML, Brussow H: Phage-host interaction: an ecological perspective. J Bacterio/ 2004, 186:3677-3686

6. Kohanski MA, Dwyer DJ, Hayete B, Lawrence CA, Collins JJ: A common mechanism of cellular death induced by bactericidal antibiotics. Cell 2007, 130:797-810.

7. Maiques E, Ubeda C, Campoy S, Salvador N, Lasa I, Novick RP, Barbe J, Penades $J R$ : Beta-lactam antibiotics induce the SOS response and horizontal transfer of virulence factors in Staphylococcus aureus. J Bacteriol 2006, 188:2726-2729

8. Zhang X, McDaniel AD, Wolf LE, Keusch GT, Waldor MK, Acheson DW: Quinolone antibiotics induce Shiga toxin-encoding bacteriophages, toxin production, and death in mice. J Infect Dis 2000, 181:664-670.
9. Stecher B, Denzler R, Maier L, Bernet F, Sanders MJ, Pickard DJ, Barthel M, Westendorf AM, Krogfelt KA, Walker AW, Ackermann M, Dobrindt U, Thomson NR, Hardt WD: Gut inflammation can boost horizontal gene transfer between pathogenic and commensal Enterobacteriaceae. Proc Natl Acad SCiUSA 2012, 109:1269-1274

10. Modi SR, Lee HH, Spina CS, Collins JJ: Antibiotic treatment expands the resistance reservoir and ecological network of the phage metagenome. Nature 2013, 499:219-222.

doi:10.1186/gb-2013-14-7-127

Cite this article as: Sun CL, Relman DA: Microbiota's 'little helpers':

bacteriophages and antibiotic-associated responses in the gut microbiome. Genome Biology 2013, 14:127. 